\title{
LITHOLAPAXY: ITS EVOLUTION, INDICATIONS, AND CONTRAINDICATIONS, WITH A RECORD OF CASES.
}

\author{
By R. J. WILLAN, NEWCASTLE-UPON-TyNE.
}

The stages in the evolution of the lithotrite are briefly traced up to $\mathrm{I} 878$, when the operation of litholapaxy was introduced by Bigelow.

$A$ resume is given of the symptoms and signs of a vesical calculus, together with the indications and contraindications for litholapaxy.

Especial stress is laid upon the necessity of systematically using the cystoscope; (I) Before operation, to ascertain if the case is suitable for litholapaxy; (2) After operation, to prove that no fragment remains in the bladder.

Some conclusions are deduced from twenty-two consecutive cases successfully treated by this method.

\section{HISTORICAL ACCOUNT.}

I Do not propose to go deeply into the ancient history of lithotrity, but merely to trace the stages in the evolution of the lithotrite, or rather the evolution of the process of reducing a stone in the bladder to fragments by the crushing operation.

Period before January 13, 1824.-There is some doubt as to who invented lithotrity; but breaking a large stone before its extraction was evidently practised by prehistoric surgeons, for Celsus, in his treatise "De Remedica," recommends the proceeding, though he failed to describe his method of accomplishment. Again, Sucruta, in the "Indian Treatise of Medicine," shows that he clearly recognized the importance of extracting every portion of the stone, for he says : "The doctor must take great pains neither to break nor to chip the stone, for if a single fragment remains, no matter how small, it would gradually'enlarge. This is why the surgeon must seize the whole stone and nothing but the stone."

From a phrase taken from an Arab author, Azzahravi, A.D. I5I9, it is suggested that lithotrity was known to this people. The following are the words which led to this suggestion: "Let an instrument which they call Mashaba Rebilia be taken and gently introduced into the passage, crush the stone in the middle of the bladder, and should it be soft, it will break and come out." Leroy d'Etoilles denied that the Arabs either practised or knew lithotrity; as to whether they did or not, depends upon the translation of the words "Mashaba Rebilia," for there is no record of how their knowledge was acquired, nor of the instruments they used. Probably the explanation is that the Arabs optimistically foreshadowed the possibility of lithotrity, although they were unable to carry the theory into practice. In the same way, the future possibility of travelling by aviation was recognized long ago, but it is only during the last few years that it has become an accomplished fact. If the Arabs really did practise lithotrity, the process was rediscovered anew centuries later.

Sanctorius, in 1626 , invented a hollow tube with three-pronged forceps which 
transmitted a stylet with an arrow-shaped head. Haller thought that this arrow-head was to break the stone, whereas it was merely to close the prongs of the forceps. Sanctorius, without suspecting it, therefore conceived a method of lithotrity, which, however, he considered inapplicable. Unfortunately, the drawing of the instrument invented by Sanctorius is quite unintelligible, and it is impossible, from an examination of it, to have any idea of the mechanism which it represents.

Fabrice de Hilden manufactured pronged forceps to extract the whole stone from the urethra, but he did not think of applying it to the bladder.

In the literature, reference is made to at least two persons who operated upon themselves: (I) a Monk of Citeaux, who crushed a stone by inserting a hollow tube into the bladder; this transmitted an iron stem which he struck with a hammer, and splintered the stone; (2) Major Martin, who was also alleged to have operated through a hollow tube, reduced the stone to fragments by means of a file. The latter gentleman at any rate did not cure himself, for it is recorded that he died of stone in Calcutta.

Probably Leroy's statement is correct, that, "Up to the beginning of this (the eighteenth) century, no instrument or apparatus by means of which one could crush the stone in the bladder had been described by any author; no instance of mechanical destruction of the stone-the surgeon operating-was known."

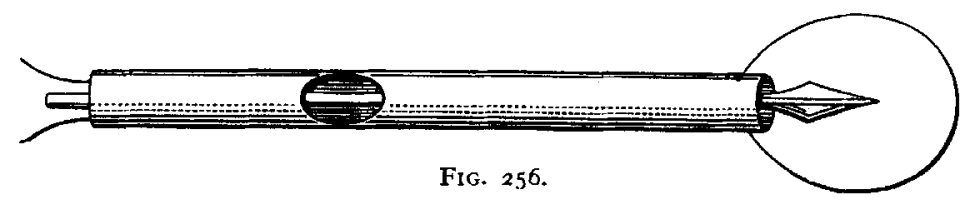

Gruithuisen, in $18 \mathrm{I} 3$, was probably the first really to practise lithotrity. His instrument consisted of a straight hollow tube which conveyed a gimlet ending in a spiked crown, or lance-shaped point (Fig. 256). In the space between the gimlet and the outer hollow tube passed a brass wire, the loop of which could be projected by the operator into the bladder to lasso the stone. When the stone had been snared, the wire held it firmly against the open mouth of the hollow tube, while the operator rotated the gimlet, which perforated the calculus. It was a dangerous instrument, as the bladder was at the mercy of the spike, but Gruithuisen at any rate showed that the bladder cavity could be reached by a probe of considerable size.

In 1822 , Leroy d'Etoilles and Amussat each demonstrated an instrument for the mechanical destruction of a vesical calculus, before the Academy of Surgery in Paris, but each instrument differed widely in principle.

Leroy aimed at destroying the stone by perforating it. He called his instrument the lithoprione; it consisted of two hollow tubes, between which slid four pieces of flattened flexible wire, the inner ends of these being fixed together by a button, making a cage at the bladder end of the instrument. When the stone had been snared in the meshes of the "cage," the wires were pulled taut to fix it, when a gimlet was introduced down the lumen of the inner tube, rotation of this gimlet boring a hole in the stone. Unknowingly, Leroy had rediscovered Gruithuisen's invention. 


\section{THE BRITISH JOURNAL OF SURGERY}

Amussat's instrument pulverized the stone by combined pressure and friction. It consisted of a straight hollow tube whose lumen acted as a sheath for two strong iron rods (Fig. 257). One end of each rod consisted of half a cylinder, this portion of the rods being projected into the bladder, the flat apposing surface of each being fitted with teeth. The other extremity of each of these rods was fitted with several angular teeth, over which a catch attached to the end of the hollow tube slipped; this catch prevented any backward movement

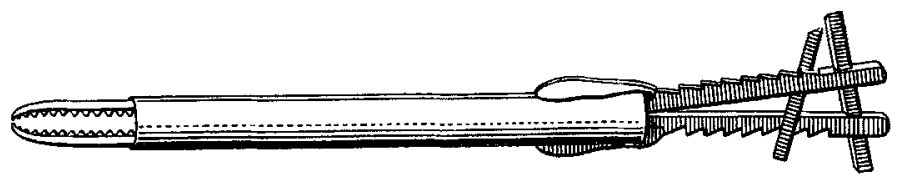

Fig. 2.57 .

of the rod, similar to the modern "ratchet." These two blades were to be projected into the bladder and the stone caught, when each blade was alternately withdrawn to the extent of one tooth. The grasped stone was thus subjected to combined friction and ever-increasing pressure.

Amussat's instrument, though a straight one, was the pioneer of the presentday lithotrite. Unfortunately for mankind, it was not strong enough to crush the stone in the bladder of the cadaver before the Commission appointed by the Academy of Surgery. As Leroy's method of perforation was, notwithstanding its imperfections, fairly successful at this demonstration, it remained in vogue for many years, until it was finally displaced, first by Heurteloup's, and then by Jacobson's modification of Amussat's invention.

January 13, 1824. - The Birthday of Practical Lithotrity. Upon this date, at the Necker Hospital, Paris, the first public demonstration of the operation of lithotrity was given, the operator being Civiale. The instrument used was a straight onc.

Civiale, in the previous year, had invented an instrument (Fig. 258), consisting of two metal cylinders $A$ and $B$, one fitting inside the other. The smaller tube

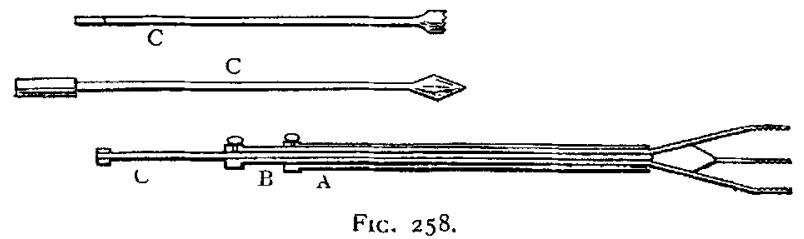

had three branches fixed on to its distal end by means of hinges. When the stone had been percussed, the smaller tube was projected into the bladder, its branches being thereby separated from each other; and the action of withdrawing the smaller cylinder into the larger one fixed the stone, when it was perforated by a gimlet $C$. This trilobed pincer was very imperfect, chiefly on account of want of elasticity of the jointed branches.

After Civiale's public demonstration, there was the famous long and bitter controversy with Leroy, for the latter alleged that Civiale had filched his instrument, which he maintained was the first to make lithotrity practicable. 
Their method (i.e., the instrument fixed and perforated the stone) was identical, though they differed in detail.

January 24, 1824 to 1878.- System of Multiple Perforations of the Stone.-For this method a drill, a drill bow, and a vice (at the bladder end of the instrument) were used. The stone was grasped by the vice, bored, released, and grasped anew. When a sufficient number of holes had bcen bored, the stone was easily crushed by the vice. Civiale practised this consistently for twelve years. It was not popular with the patient, because seizing the stone was a most difficult and painful proceeding, though Civiale denied this.

Excentric Lithotrity, i.e., the Destruction of the Stone from the Centre of the Stone to the Periphery.- On account of the pain produced by the system of multiple perforations, surgeons, other than Civiale, found it impossible to carry out the operation by this method, owing to the necessity of releasing and re-grasping the stone. It was realized that the stone must be pulverized without having constantly to release and recapture it; therefore Heurteloup popularized excentric lithotrity. His straight hollow forceps held the stone, while his hook-shaped drill with adjustable wings (introduced down the lumen of the forceps) did the hollowing out. The objection to this method was the difficulty in removing the shell of the hollowed-out stone.

Concentric Lithotrity (i.e., the pulverizing of the Stone from the Periphery towards the Centre).-Rigal also grasped the stone with forceps, but he rubbed the stone from the circumference to the centre by making the stone rotate in the bladder against the roughened branches of his instrument, which acted as a grater. It proved too painful a method to be practised.

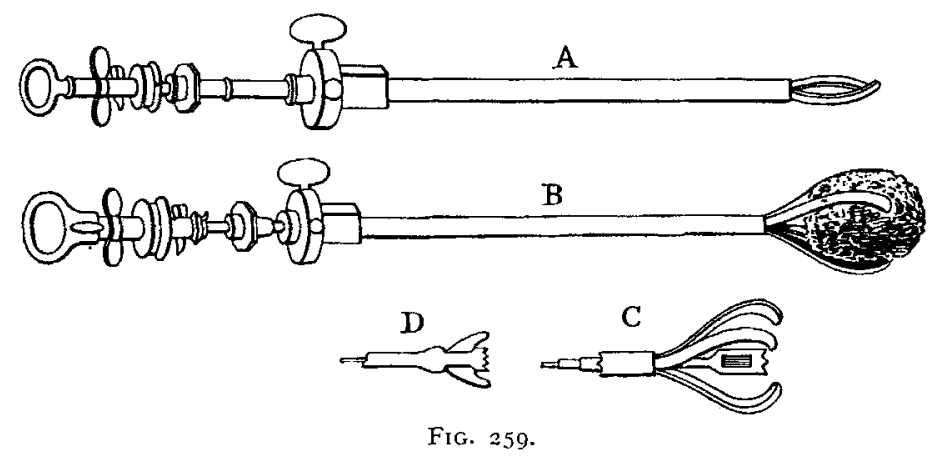

Splintering may be regarded as a transition stage between progressive pressure and crushing. The stone was grasped by prongs on the end of a hollow straight tube (Fig. 259, $A$ and $B$ ), which was large enough to accommodate a drill. On the sides of the flattened extremity of this drill were two concealed wings capable of expansion by mean of a screw device (Fig. 259, C and D). After a hole had been made in the calculus, the drill was placed in the middle of the stone, and the wings were made to project in an outward dircction, when the stone was disrupted by the expanding force.

Division of the Stone by a Screw.-Weiss, of London, introduced a curved beaked instrument. It consisted of two parts, one sliding in a groove in. the 
other; between the beaks was a small saw meant to cut the stone by a backward and forward movement.

Crushing the Stone.-Gruithuisen invented an instrument consisting of a strong metal rod, having at one end two diverging branches, which were sharp along the apposing edges, like the blades of a pair of scissors. This rod was drawn into an external straight hollow tube, which determined the approach of the diverging blades to each other. The stone was thus cut, and also tended to be crushed.

Crushing by "Snappers" and a Lever; Contraction and Friction Combined.Heurteloup recognized the value of Amussat's instrument and modified it, but

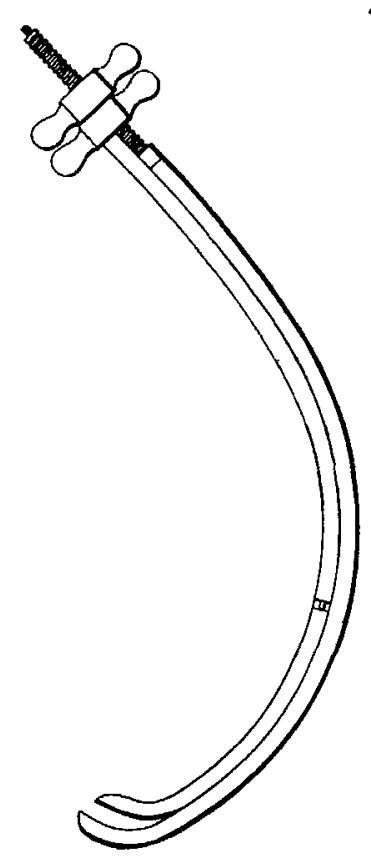

FIG. 260 .

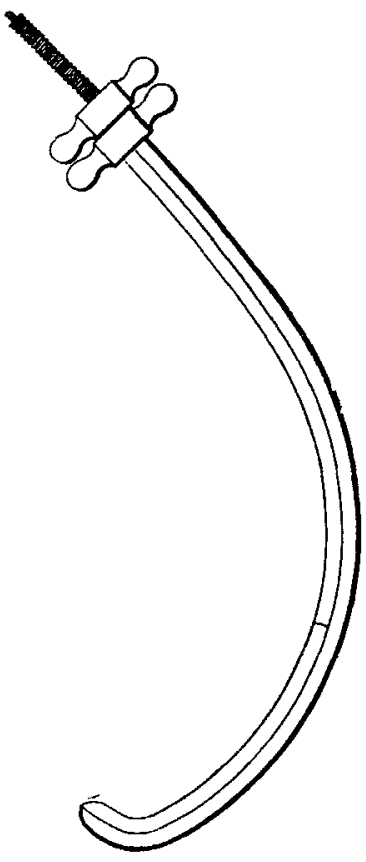

FIG. 26 I. his modification was displaced by Jacobson's further modifcation, which was an articulated stone-crusher.

Crushing by the Action of a Screw and Vice.-As applied to the bladder, Leroy in 1823 was the first to think of using the screw and vice in lithotrity. In 1825 Leroy and Retore made an instrument consisting of two curved blades sliding against each other in a groove. The bladder-end of each blade terminated in a beak; the approximation or divergence of these beaks was accomplished like the action of a vice through the medium of a screw at the opposite, or outer, end of the instrument. (Fig. 260 shows the instrument open, while Fig. 26I shows it closed.) The stone was to be crushed between the beaks.

This was a pioneer instrument, but it broke, and was never used. Leroy did not recognize the value of it, and continued to pulverize calculi by drills.

However, the instrument which was the first to show practically the strength of crushing by the action of the vice and screw was Jacobson's articulated stonecrusher, brought out in I829. When closed it resembled a large sound slightly curved, and it consisted of an outer silver sheath enclosing a steel rod. The rod was divided into two parts, an anterior immovable one and a posterior movable portion; these were united at their vesical end by a hinge. Fig. 262 shows (a) the sheath or body of the instrument; $(b)$ the immovable branch; $(c c c)$ are the joints of the movable branch; $(f)$ is the point of support for the female screw. To crush a stone, the movable portion of the rod was projected into the bladder; this was withdrawn by means of the screw action of the vice after the calculus had been snared, when the stone was crushed. 
It was criticized as being of insufficient strength owing to the numerous articulations. The jointed stone-crusher was the beginning of a new era in lithotrity. Jacobson's instrument, however, was regarded as inferior to Heurteloup's grooved stone-crusher or percussor.
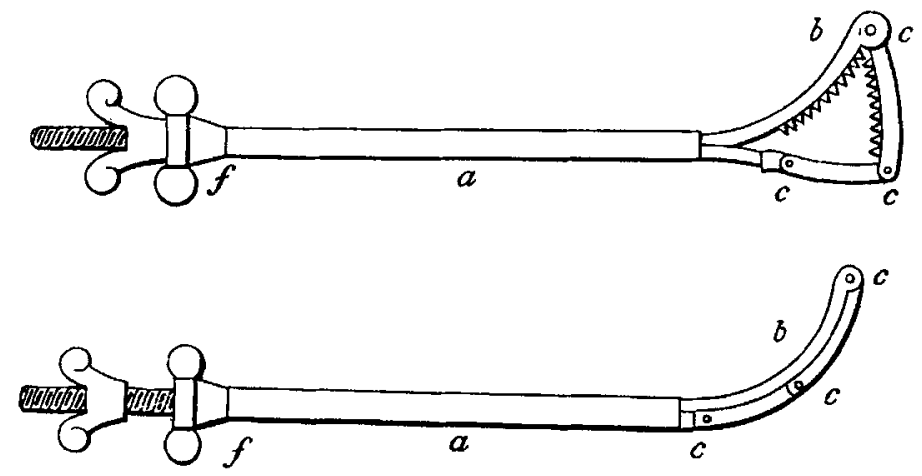

Fic. 262 .

Percussion and the Percussor.-Leroy's, Weiss's and Heurteloup's instruments were similar in being curved and in having two blades sliding against each other in a groove, i.e., they fixed the calculus in a vice. Heurteloup, in 1823 , described his instrument, which deserves special mention, as it was the first to be used satisfactorily for lithotrity: moreover, it is the one, with some modifications, which is in use to-day.

The instrument when closed resembled a curved catheter, and consisted of two blades, a larger female, in which slid the smaller male one. The female blade had a square enlargement on the outer end, and this was firmly fixed into a vice, which was a fixture on a special operation-table. When the curved extremities of the inner end of the instrument were separated, a gap was left; the stone was manipulated into this gap. After the stone had been secured, the
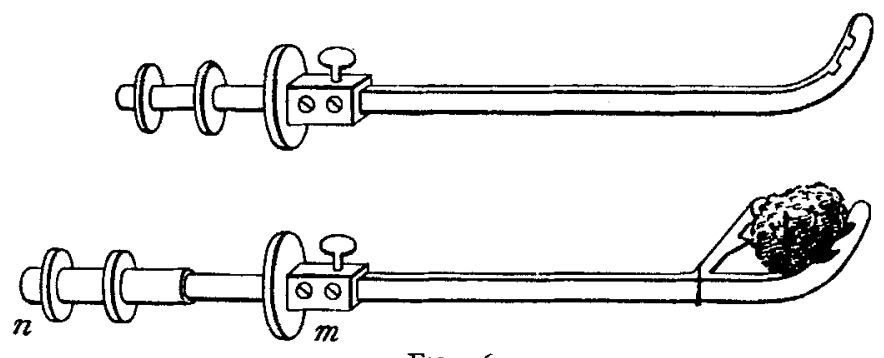

Fro. 263 .

female blade was fixed in the vice attached to the operation-table, and the outer end of the male blade was struck sharply with a hammer, which pulverized the calculus. Fig. 263 shows the instrument, open and closed; $m$ is the square on the female blade, which was held in the vice fixed to the operation-table; percussion was applied at the point $n$. 
Crushing by Pressure of the Hands.-Percussion was found to be inconvenient, as it hurt the patient, whose slightest movement injured the bladder; moreover, it took time to fit the instrument to the operation-table vice each time the stone was seized. Attempts were therefore made to crush calculi by simple pressure of the hands; this method was discontinued, as there was too much risk of injury to the bladder when the blades approached each other too abruptly and jerkily.

The next advance was in 1832 , when Touzay conceived the idea of fitting a screw to the outer end of the lithotrite, so that the movable blade could be

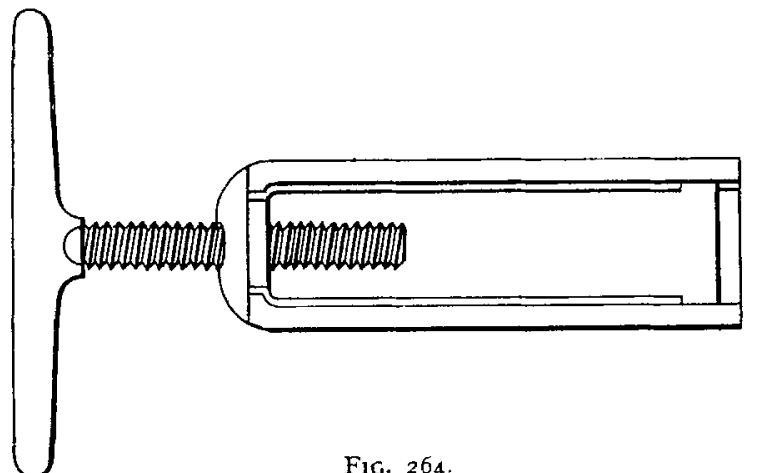

F1G. 264 . evenly projected towards the male blade. This compressor, or screw, was detachable (Fig. 264), and was fitted to the instrument after the stone had been grasped. Later, the compressor became fixed and part of the instrument, and the present system of locking and unlocking the two blades at will was invented.

After this (I846), surgery went mad inventing new

instruments for "perineal lithotrity," new dilators for the prostatic urethra being the most fashionable inventions. Didot had previously suggested perineal lithotrity, but did not practise it, while Bouisson put this theory into execution.

Evacuation of the Crushed Fragments.-In I843, Cornay, of Rochefort, invented the first aspirator. The essential parts of this apparatus were: (I) A slightly curved sound open at its end, (2) a glass flask, or receiver, (3) a long flexible tube connecting the flask to the sound, and (4) an aspirating pump.

In I866 Clover's apparatus appeared. The receiver was a pear-shaped vessel made of india-rubber. Its chief advantage was a special method of preventing the fragments of the stone returning to the bladder.

Although neither of these aspirators was efficient, they must be regarded as forerunners of Bigelow's evacuator.

Litholapaxy, 1878.- - From the time of Heurteloup up to the time of Bigelow the science of lithotrity had advanced slowly. In 1878 Bigelow introduced the operation of litholapaxy, the name being derived from $\lambda_{\iota} \theta$ os $=$ "a stone," and $\lambda a \pi a \xi_{\iota c}=$ " evacuation." He crushed the stone and evacuated the fragments at one sitting.

His predecessors lacked two things which prevented them from arriving at perfection in lithotrity : firstly, the use of anæsthetics; and secondly, instruments by which they could empty the bladder of the stone fragments. Bigelow's invention of an efficient evacuator, with the employment of anæsthesia, completely revolutionized the operation for stone, and all credit is due to this American for his ingenuity. Fig. 265 shows his lithotrite and Fig. 266 his evacuator. 


\section{RÉSUMÉ OF SYMPTOMS AND SIGNS.}

The symptoms produced by a vesical calculus are usually more severe in children than in adults. This phenomenon is explained by the child's bladder being an abdominal organ; it consequently lies in a vertical position, and the stone therefore tends to engage constantly in the internal meatus when the child is in the upright posture. In an elderly male, the stone may lie in a pouch behind the prostate gland, which effectually prevents it from engaging in the internal urinary meatus.

Symptoms of Uncomplicated Vesical Calculus. - The patient usually gives a history of :-

Increased frequency of micturition while walking about, but with no deviation from the normal in this respect while he is resting.

Pain along the whole length of the urethra, or at the end of the penis, either towards the termination of micturition, or while walking over rough ground. Severe pain after micturition is often felt in the perineum or in the anus itself. The pain is due to mechanical irritation of the nerve plexus on the sensitive trigone of the bladder.

The patient will often volunteer the statement that stepping on or off the street kerbstone will produce a spasm of pain, more or less severe.

The stream is normal in calibre and force, but there may be sudden stoppages of micturition followed by a return to the normal, with any slight alteration in his position. The severe straining which FIG. 265 .

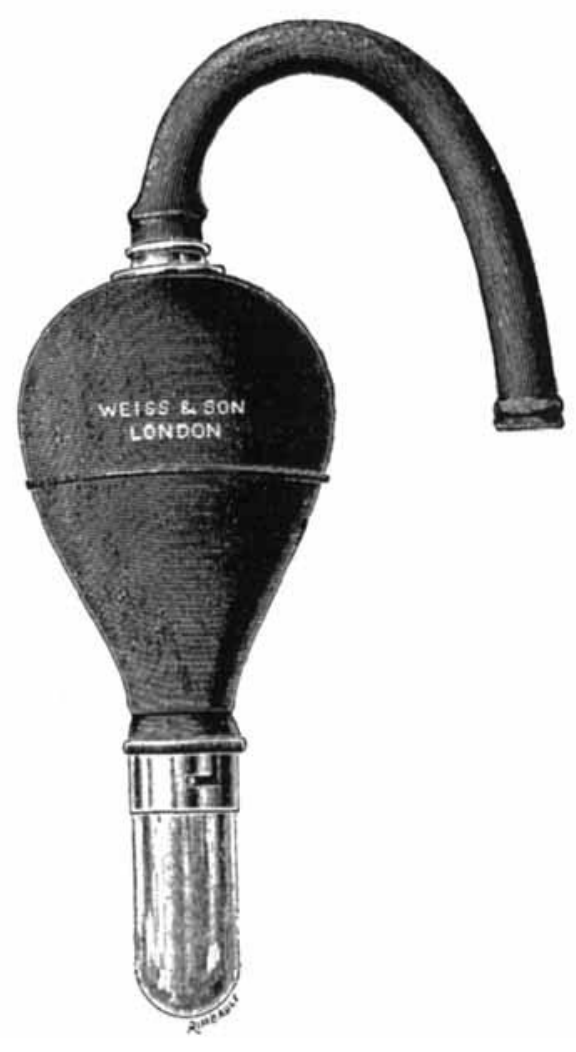

Fic. 266. ensues when the calculus is engaged in the internal meatus sometimes produces incontinence of fæces during micturition, and that patient will consequently have a tendency to hæmorrhoids.

Bleeding towards the end of micturition, especially after severe exercise (such as horse-riding), walking over rough ground, or jolting in a moving road vehicle which is devoid of springs. 


\section{THE BRITISH JOURNAL OF SURGERY}

A history is often given that a child is constantly pulling at his penis to relieve pain felt at the end of the organ, this pain being due to the irritation of the calculus lying on the bladder trigone. As a result of this constant "milking," the penis is well developed.

Symptoms of a Vesical Calculus with Cystitis.-If a vesical calculus is complicated with cystitis which is acute or chronic, then it is often extremely difficult to make a correct diagnosis without the aid of the cystoscope. The symptoms are then like those of a tuberculous cystitis, i.e., in addition to the ordinary symptoms of stone, there are increased frequency of micturition day and night, with more or less pain in the hypogastrium.

Signs.-It is always advisable to ask the patient to micturate, and for the surgeon to see for himself if there are any sudden stoppages, any hæmaturia, the degree of pain, etc. Although uncommon, it is possible for a vesical calculus to be felt on a rectal examination. The urine should be carefully examined for traces of blood and pus.

Priapism is very often a definite sign of the presence of vesical calculus. I recently saw a child aged six weeks where this sign was well marked, and who had a small stone in his bladder.

One can never be quite certain that a stone is present until it is actually seen with the cystoscope, for although radiography is of great use it is not conclusive. Abnormal shadows in the pelvis may be entirely outside the bladder or ureter; again, a stone which is formed of pure uric acid may not throw a shadow:

The bladder sound is rapidly falling into disuse, as it can be very misleading, especially in children where the spine of the ischium may be percussed with the end of the sound, which has on occasions been mistaken for a vesical calculus.

\section{INDICATIONS FOR LITHOLAPAXY.}

General Considerations.-In that unique stone number of the Indian Medical Gazette (August, Igoo), where not only the statistics, but also the operative experience, of famous lithotritists are given, the general consensus of opinion is that litholapaxy is certainly the best operation for the removal of a vesical calculus, and that the knife should only be used as a last resort.

In skilled hands and in carefully selected cases it is a very safe operation. It must be remembered, however, that crushing a stone in a child's bladder is a much more difficult undertaking than in an adult. Therefore, a novice will be well advised if, in his earlier cases, he practises litholapaxy exclusively upon adults.

If a patient has had one calculus, it is more than possible that he will have a further one; the first suprapubic lithotomy is a very easy matter, but not so the second and succeeding operations, where the peritoneum is drawn downwards by the cicatricial contraction from the previous operation; opening up of the peritoneum, especially where there is a cystitis, runs a very serious risk of general septic peritonitis.

Indications.-The operator's armamentarium must include a suitable collection of lithotrites, with aspirators and numerous cannulæ. Vesical calculi which are to be successfully_removed by the operation of litholapaxy must be 
selected. In this selection the surgeon is largely dependent upon the cystoscope, which should eliminate the complications of :-

I. A marked enlargement of the prostate gland.

2. An encysted vesical calculus.

3. Sacculations of the bladder which cannot be smoothed out by further distention of the bladder.

4. A malignant growth of the bladder.

In the absence of these complications, and provided the patient's kidneys are healthy, a calculus of large size can be safely removed by litholapaxy. A cystitis, however acute, is no contraindication to the crushing, for the inflammation will subside immediately the causative irritant (the calculus) is removed.

Disability.-After a litholapaxy, it is uncommon for a patient not to be able to return to work within seven days after operation.

After a suprapubic lithotomy, he will be lucky if he has sufficiently recovered his strength to do full work again before six weeks have elapsed. If he returns to work too soon, there is a real danger of the scar yielding and of his getting a post-operative hernia. Moreover, even when there is no apparent obstruction - in the urethra, a suprapubic sinus occasionally persists for months.

With perineal lithotomy, permanent incontinence of urine sometimes results, owing to damage to the bladder sphincters, either by the scalpel or by the tearing out of the stone.

Recurrence of the Calculus.-A recurrence of the calculus is given as a contraindication for litholapaxy, but there would only be a recurrence if a fragment of stone is left. As it is my invariable practice always to cystoscope the patient, either immediately after the crushing or before the patient leaves my care, I can eliminate the possibility of this happening. In the ordinary cases there is no bleeding, and one is able to do this immediately after the litholapaxy.

If hæmorrhage prevents the use of the cystoscope at the conclusion of the operation, I search the bladder by means of the aspirator and cannula as recommended by Freyer. ${ }^{1}$ The operator having placed his ear on the patient's hypogastrium, a few compressions of the aspirator are made, and the lotion is propelled along the cannula into the bladder. The returning fluid causes any calcareous fragment, too large to pass, to impinge against the eye of the cannula, which produces an audible " click."

I consider that a recurrence of the stone is quite as common after suprapubic lithotomy as after litholapaxy. Very often vesical calculi only give marked trouble when cystitis has supervened; if suprapubic lithotomy has been performed where the bladder is septic, it is always wise to employ drainage, and it is wonderfully easy for a piece of ligature or dressing to fall into the bladder and form a nucleus for a further stone. Moreover, if care is not taken after a suprapubic lithotomy to keep the urine acid, especially in the presence of sepsis, a phosphatic deposit adheres to the drainage tube, and fragments of this fall into the bladder, to form the nucleus of another calculus.

\section{CONTRAINDICATIONS FOR IITHOLAPAXY.}

Skill in Manipulation.-A surgeon who is not accustomed to the operation of litholapaxy will be wise if he does not attempt to remove the calculus by this operation; in unskilled hands suprapubic lithotomy is safer. 


\section{THE BRITISH JOURNAL OF SURGERY}

Enlarged Prostate Gland.-With a marked enlargement of the prostate gland, especially of the middle lobe, I consider it is unsafe to attempt to crush a vesical calculus. It is difficult to catch the stone owing to its being in a postprostatic pouch; severe bleeding may ensue from damage to a rapidly-growing prostate; moreover, a rupture of the bladder by the lithotrite (an accident which one occasionally hears of) is probably always due to an attempt to crush the stone in the presence of a marked enlargement of the prostate. It is, of course, only possible to accurately diagnose an enlarged middle lobe of the prostate gland by seeing it through a cystoscope.

Encysted Calculus, i.e., a calculus which is encysted in a sacculus in the bladder wall, a portion only of the stone being in the cavity of the bladder. This will be seen in a routine cystoscopic examination.

Sacculation of the Bladder.- - If a stone is crushed in a sacculated. bladder, fragments of the stone are apt to fall into these sacculi, and not only form the nucleus of an encysted calculus, but they may set up a perivesical abscess, due to the sharp fragments perforating the thin wall of the sacculus. In some instances it is possible to smooth out shallow sacculi by further distention of the bladder; in such a case it is quite safe to employ litholapaxy.

Size of the Stone.-A stone of too large a size has been given as a contraindication; but this, I think, depends entirely on the patient's general condition, and that again depends upon the state of the kidneys. If a patient is in good health, I think even a large stone can be removed by nibbling away at its sides. until it is small enough to be caught in the jaws of the lithotrite. Freyer ${ }^{2}$ defines a "large stone" as one weighing more than two ounces.

Consistence of the Calculus.-A stone of very hard consistence is. especially given by Indian lithotritists as a contraindication for litholapaxy. These hard stones are probably more often met with in India than in this country.

Stricture of the Urethra.-A passable stricture of the urethra is not a contraindication unless there are further complications: for example, a stricture with perineal fistulæ. In this case it is wisest to remove the stone by a cutting operation.

A Foreign Body as the Nucleus of a Stone (e.g., a fragment of catheter, bougie, etc.). If it is known that a foreign body (which is too large to evacuate) forms the nucleus of a stone, it is best to remove the calculus by suprapubic lithotomy.

Age.-Male children under the age of three should have the stone removed. by a suprapubic lithotomy, although it entirely depends on the size of the urethra, which is surprisingly large in children.

Stone in the Prostatic Urethra.-A stone lodged in the prostatic urethra. which could not be easily pushed back into the bladder should be removed by perineal lithotomy.

Pyonephrosis or Pyelonephritis.-Where renal sepsis is present, it is always wise to do a suprapubic lithotomy in order that free drainage may be established, thereby resting the kidneys as much as possible. A study of the statistics in the Indian Medical Gazette for August, I9oo, clearly shows that renal disease is very largely responsible for the mortality in litholapaxy 
The symptoms of bilateral renal pain, intense thirst, frontal headaches on awakening in the morning, and loss of flesh, are invaluable evidence of renal insufficiency.

Malignant Growth of the Bladder.-A calculus with a vesical malignant growth is uncommon; when present, it should be removed by suprapubic lithotomy.

\section{AUTHOR'S SERIES OF CASES.}

Litholapaxy (including lithotrity) is not a common operation. In a search of the case notes of the Royal Victoria Infirmary, Newcastle-upon-Tyne, up to December 3I, I9I2, I could only find 26 cases where a vesical calculus was removed by the crushing operation. It may be performed in two ways: (I) By urethral litholapaxy, i.e., inserting the lithotrite through the intact natural passages; and (2) By perineal litholapaxy, i.e., making an incision into the urethra in the perineum and introducing the lithotrite through this incision into the bladder, via the prostatic urethra. I have no experience of the latter method, and my remarks in this monograph refer entirely to the former procedure.

Each case was carefully selected; that is, a careful cystoscopic examination was made before deciding upon the crushing operation. Moreover, in each instance, before the patient finally left my care, I satisfied myself with the cystoscope that no fragment remained in the bladder.

Up to date I have successfully performed litholapaxy upon twenty-two cases, two of these being females. In a further case not included in the series, I crushed a portion of a calculus weighing I2 ounces, ${ }^{3}$ but had to complete its removal by suprapubic lithotomy, owing to the bad general condition of the patient, a male aged 6o. Had its removal been accomplished entirely by litholapaxy, it would have equalled the record calculus crushed by Milton, of Cairo.

The following are the weights of some of the large vesical calculi removed through the natural passages:-

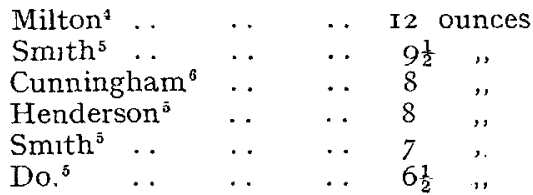

\begin{tabular}{|c|c|c|c|}
\hline Freyer ${ }^{7}$. & . . & & \\
\hline Smith ${ }^{3}$ & & & 6 \\
\hline Do. ${ }^{\circ}$ & & & $5 ?$ \\
\hline At Jaupur & Hospital & & 3 \\
\hline Davidson & 1 & & $3 \frac{1}{3}$ \\
\hline
\end{tabular}

The largest stone I have crushed weighed exactly 2 ounces, a further one being just short of this. Thirteen of the series exceeded I drachm; the remaining nine were under this weight, the smallest being 5 grains.

The youngest case was 5 years old, and the oldest 72 , the average age being 42 .

Thirteen of the cases were $x$-rayed. This examination gave a negative result in two instances, and in each of these two cases a calculus of pure uricacid consistency was subsequently removed.

Only a small proportion of the crushed calculi have been examined chemically, therefore I have not ventured to state the consistence of the calculi. 


\section{THE BRITISH JOURNAL OF SURGERY}

Every case I have operated upon by this method has made an uneventful recovery.

For the historical account I am chiefly indebted to Gouette's "Essai Historique sur la Lithothritie," I893; and to Leroy d'Etoille's " Histoire de la Lithothrite," I839. The bulk of the illustrations are reproduced from the latter monograph, and I have also to thank Mr. P. J. Freyer for his kindness in allowing me to reproduce illustrations 265 and 266 from his "Modern Treatment of Stone."

\section{REFERENCES.}

1 Freyer, Ind. Med. Gaz., I884.

2 Freyer, Brit. Med. Jour., I 896 , ii.

${ }^{3}$ WILlaN, Northumberland and Durham Med. Jour., 19r3. Sept.

${ }^{4}$ Lancet, 1896 , i.

5 Ind. Med. Gaz., 1900, Aug.

${ }^{6}$ Brit. Med. Jour., I89r, i, 1008.

7 Freyer, Modern Treatment of Sione, 1896 . 